\title{
Chronic Back Pain Is Associated with Decreased Prefrontal and Thalamic Gray Matter Density
}

\author{
A. Vania Apkarian, ${ }^{1}$ Yamaya Sosa, ${ }^{1}$ Sreepadma Sonty, ${ }^{2}$ Robert M. Levy, ${ }^{3}$ R. Norman Harden, ${ }^{5}$ Todd B. Parrish ${ }^{4}$ and \\ Darren R. Gitelman ${ }^{2,4}$ \\ ${ }^{1}$ Department of Physiology and Institute of Neuroscience, and Departments of ${ }^{2}$ Neurology, ${ }^{3}$ Neurosurgery, and ${ }^{4}$ Radiology, and ${ }^{5}$ Rehabilitation Institute of \\ Chicago, Northwestern University Feinberg School of Medicine, Chicago, Illinois 60611
}

The role of the brain in chronic pain conditions remains speculative. We compared brain morphology of 26 chronic back pain (CBP) patients to matched control subjects, using magnetic resonance imaging brain scan data and automated analysis techniques. CBP patients were divided into neuropathic, exhibiting pain because of sciatic nerve damage, and non-neuropathic groups. Pain-related characteristics were correlated to morphometric measures. Neocortical gray matter volume was compared after skull normalization. Patients with CBP showed 5-11\% less neocortical gray matter volume than control subjects. The magnitude of this decrease is equivalent to the gray matter volume lost in 10-20 years of normal aging. The decreased volume was related to pain duration, indicating a $1.3 \mathrm{~cm}^{3}$ loss of gray matter for every year of chronic pain. Regional gray matter density in $17 \mathrm{CBP}$ patients was compared with matched controls using voxel-based morphometry and nonparametric statistics. Gray matter density was reduced in bilateral dorsolateral prefrontal cortex and right thalamus and was strongly related to pain characteristics in a pattern distinct for neuropathic and non-neuropathic CBP. Our results imply that CBP is accompanied by brain atrophy and suggest that the pathophysiology of chronic pain includes thalamocortical processes.

Key words: chronic pain; morphometry; frontal cortex; thalamus; neuropathic back pain; aging

\section{Introduction}

Ten percent of adults suffer from severe chronic pain (Harstall and Ospina, 2003). Back problems constitute $25 \%$ of all disabling occupational injuries and are the fifth most common reason for visits to the clinic; in $85 \%$ of such conditions, no definitive diagnosis can be made (Cavanaugh and Weinstein, 1994; Deyo, 1998). The impact of chronic pain on the nervous system has been studied primarily in animal models (Woolf and Salter, 2000; Hunt and Mantyh, 2001; Julius and Basbaum, 2001). Such studies highlight reorganization of nociceptive coding by peripheral afferents and spinal cord neurons and provide evidence for apoptosis of spinal cord cells (Whiteside and Munglani, 2001; Moore et al., 2002; de Novellis et al., 2004). Many of these changes are commonly observed in both inflammatory (caused by tissue injury) and neuropathic (caused by neuronal injury) pain, whereas others are specific to one type of pain or the other. Moreover, these subtypes of chronic pain exhibit distinct clinical characteristics (Dworkin, 2002). Although chronic pain greatly diminishes quality of life and increases anxiety and depression (Riley et al.,

\footnotetext{
Received June 25, 2004; revised 0ct. 11, 2004; accepted 0ct. 12, 2004.

This work was supported by National Institute of Neurological Disorders and Stroke Grant NS-35115 to A.V.A. and National Institute on Aging Grant K23 AG-00940 to D.R.G. from the National Institutes of Health. We thank all patients and volunteers for participating in this study. We thank the Cognitive Brain Mapping Group, especially $P$. Reber, for providing MRI data of volunteers, and M. M. Mesulam, D. Small, D. Pelli, and S. A. Khan for commenting on previous versions of this manuscript.

Correspondence should be addressed to A. Vania Apkarian, Department of Physiology, Northwestern University Medical School, 5-120 Ward Building, 303 East Chicago Avenue, Chicago, IL 60611. E-mail: a-apkarian@northwestern.edu. D0I:10.1523/JNEUROSCI.2541-04.2004

Copyright $\odot 2004$ Society for Neuroscience $\quad$ 0270-6474/04/2410410-06\$15.00/0
}

2001; Dworkin, 2002), it is assumed that the cerebral cortex passively reflects spinal changes and reverts to its normal state after cessation of chronic pain (Price, 2000; Mendell and Sahenk, 2003). Our studies show that chronic back pain (CBP) (sustained for $>6$ months) is accompanied by abnormal brain chemistry (Grachev et al., 2000), mainly a reduction in the $N$-acetyl-aspartate-creatine ratio in the prefrontal cortex, implying neuronal loss or dysfunction in this region and reduced cognitive abilities on a task that implies abnormal prefrontal processing (Apkarian et al., 2004a). To our knowledge, no studies have yet examined brain morphometry in chronic pain conditions.

We used structural magnetic resonance imaging (MRI) brain scan data and two independent automated morphometry approaches to contrast brain morphology of CBP patients to matched normal subjects. We hypothesized that neocortical gray matter should undergo atrophy in CBP above and beyond the atrophy associated with normal aging. This hypothesis was confirmed. Moreover, given our evidence for prefrontal cortical biochemical and cognitive abnormalities, we also hypothesized that the regional analysis should point to prefrontal atrophy. Previous studies have shown decreased thalamic baseline activity or decreased thalamic responses in chronic pain conditions. Therefore, we hypothesized that the thalamus should also undergo atrophy. Because both clinical and experimental findings imply that neuropathic and non-neuropathic chronic pain conditions may have distinct underlying processes, we tested for differences between these groups. The hypothesis in this case was that neuropathic pain should have a larger impact on the brain, which was verified. 
Our results demonstrate regionally specific reduced gray matter in patients with CBP. At the whole-brain level, this reduction is related to pain duration, regionally depends on multiple painrelated characteristics, and is more severe in the neuropathic subtype. Therefore, these data present strong evidence that the pathophysiology of chronic pain includes cortical processes, and the observed changes likely constitute the physical substrate of the cognitive and behavioral properties of chronic pain.

\section{Materials and Methods}

Subjects. We compared 26 CBP patients with 26 matched normal volunteers, after obtaining informed consent. Patients fulfilled the International Association for the Study of Pain (IASP) criteria for CBP (Merskey and Bogduk, 1994) and were diagnosed in accordance with recent guidelines (Deyo and Weinstein, 2001). Diagnosis was performed by experienced clinicians (R.M.L., R.N.H.) based on history, general physical exam, and detailed neurological exam, especially sensory, motor, reflex, and gait examinations. Briefly, all CBP patients had unrelenting pain for $>1$ year, primarily localized to the lumbosacral region, including buttocks and thighs, with or without pain radiating to the leg. Some CBP patients also indicated presence of pain outside this region (for example, in the upper back); they were considered to have CBP only if the main source of pain was lumbosacral (see Fig. $3 B$ ). We did not distinguish the source of CBP, which may be caused by various etiologies, such as fracture, inflammatory joint disease, postsurgical factors, combinations of these, or idiopathic factors (Deyo and Weinstein, 2001). The clinical data indicated that 15 of 26 subjects (55\%) had musculoskeletal diagnoses, five $(20 \%)$ had pure radiculopathy, and six $(26 \%)$ had a mixture of musculoskeletal and radiculopathic pain. Pairwise matching was done for gender, age ( \pm 2 years, except in two subjects in which match was within \pm 5 years), and scan sequence. CBP patients were divided into neuropathic and non-neuropathic subtypes, based on symptoms of damage to the sciatic nerve, using IASP criteria (Merskey and Bogduk, 1994). Neuropathic CBP patients were those with significant radiculopathy, with or without the presence of musculoskeletal pain (i.e., a large component of the back pain was from unilateral leg pain $(>40 \%)]$; in some this radiated to the foot or toes and was accompanied by numbness or paresthesias or by reduced straight leg raising and motor sensory or reflex changes. In non-neuropathic CBP, the leg pain component of CBP was deemed minimal (supplemental Table 1, available at www. jneurosci.org as supplemental material).

Brain scans. We performed anatomic T1-weighted MRI brain scans using two slightly different three-dimensional fast low-angle shot sequences, "no-flow" and "fast" paradigms, on a $1.5 \mathrm{~T}$ scanner. The fast protocol uses interpolation in the slice direction. The result is a $2-\mathrm{mm}$ thick slice interpolated to a $1 \mathrm{~mm}$ thickness, imaging parameters were: repetition time (TR), $15 \mathrm{msec}$; echo time, $5.6 \mathrm{msec}$; flip angle, $20^{\circ}$; matrix, $256 \times 256$; and a field of view of $240 \mathrm{~mm}$, with $160 \mathrm{~mm}$ coverage in the slice direction. In no-flow sequence, slices are acquired at $1 \mathrm{~mm}$ slice thickness using a presaturation pulse to decrease ghosting artifacts in the temporal lobes, and imaging parameters use a TR of $22 \mathrm{msec}$.

Morphometry. The brain images were used to calculate normalized cortical gray matter volume, skull-normalized to a standard brain, excluding the cerebellum, deep gray matter, and brainstem, and used to calculate normalized lateral ventricular volumes using a mask designed for this purpose. These measures were derived by the cross-sectional version of the SIENA software, SIENAX, which uses automated brain extraction and tissue segmentation software (www.fmrib.ox.ac.uk/fsl), yielding an estimate of total brain tissue volume (Smith et al., 2002). Extracted magnetic resonance (MR) images are registered to a canonical image in standardized space to provide a spatial normalization factor. The final skull-normalized neocortical gray matter volume compensates for body-mass variations between subjects. Because SIENAX output is insensitive to scan parameters (Smith et al., 2002) and our results with no-flow scans and fast scans showed no significant differences, we combined results from both scan types.

Regional gray matter density was assessed with voxel-based morphometry (VBM) using the optimized method and statistical nonpara- metric mapping analysis (Ashburner and Friston, 2000; Good et al., 2001a). VBM was performed using SPM99 software (www.fil.ion. ucl.ac.uk/spm), and the SnPM toolbox was used for nonparametric analysis (Nichols and Holmes, 2002). The technique has been validated with independent region of interest measurements (Vargha-Khadem et al., 1998; Maguire et al., 2000; Richardson et al., 2004). Images are first normalized into a standard space and then segmented. To correct for nonlinear spatial normalization, voxel values are multiplied by the Jacobian determinants of the spatial normalization. Resultant values reflect absolute amounts (volume in arbitrary units) of gray matter. The normalized segments are smoothed with a $12 \mathrm{~mm}$ full width at half maximum Gaussian kernel in all three dimensions for parametric analysis and only within plane for nonparametric analysis. Global differences in gray matter density were treated as confounds and were removed to ensure that differences pertained to regional rather than global changes in gray matter. VBM analysis is sensitive to MR scan parameters. Our analysis indicated that results from fast scans were inferior to those obtained by no-flow scans. The no-flow scans include a saturation band inferior to the volume acquisition to reduce artifacts caused by inflowing blood. In addition, the no-flow data were acquired at a $1 \mathrm{~mm}$ slice thickness, whereas the fast scans were acquired at $2 \mathrm{~mm}$ and interpolated to $1 \mathrm{~mm}$. Therefore, we only contrasted VBM results for no-flow scans. Regional changes in gray matter were assessed nonparametrically to allow rigorous cluster-based comparisons of significance. Statistical nonparametric maps were generated using a two-group, one-scan-per-subject permutation analysis (Nichols and Holmes, 2002), in which pseudo $t$ statistical analysis was performed over the entire brain. Permutation-based clusterlevel inference was also done to assess significance of cluster size. Group differences were tested against 1000 random permutations, which inherently accounts for multiple comparisons. Because we observed a thalamic decrease in gray matter density that did not pass the whole-brain multiple-comparison threshold, we repeated the nonparametric comparison after spatially limiting the analysis to data within the thalami. Regional gray matter densities were obtained from the first eigenvariate, for a $5 \mathrm{~mm}$ radius sphere at the peak of interest, which accounted for $>99 \%$ of the variance. White matter changes between CBP subjects and controls were not analyzed, because segmentation of subcortical gray matter is not very precise and can result in contaminating white matter volume estimates with gray matter and ventricular volumes. Similar contaminants may obscure estimates of ventricular volumes as measured by VBM. For this reason ventricular volumes were only measured by SIENAX, coupled with a specific mask.

Pain characteristics. Patients completed McGill pain questionnaire short forms (Melzack, 1987) to derive scalars for sensory and affective dimensions of CBP. Anxiety and depression traits were determined by questionnaires (Beck and Steer, 1993a,b), and intensity of pain was assessed on a visual analog scale $(0=$ no pain, $10=$ maximum imaginable pain) on scan day. Duration of CBP pain was measured in years, and drug consumption was calculated using a validated Medication Quantification Scale (MQS) (Middaugh et al., 1987), which reduces drugs used for different durations and doses to a single scalar. These characteristics were related to brain morphometry either by linear correlations or in multiple linear regression models with stepwise elimination (supplemental Table 2, available at www.jneurosci.org as supplemental material).

\section{Results}

\section{Cortical gray matter volume}

Skull-normalized whole-brain neocortical gray matter volume (excluding the cerebellum, deep gray matter, and brainstem; SIENAX analysis) was $528 \pm 44 \mathrm{~cm}^{3}$ (mean $\pm \mathrm{SD} ; n=26$ ) in the CBP brain and $559 \pm 42 \mathrm{~cm}^{3}(n=26)$ in controls, matched for age, sex, and scan type (Fig. $1 A$ ). The $30 \mathrm{~cm}^{3}$ difference in gray matter volume, a $5.4 \%$ decrease, was highly significant (paired $t$ test $=3.7 ; p<0.001$ ). A similar measure was derived from the VBM regional analysis: whole-brain mean gray matter density per voxel (VBM modulation analysis). This measure showed a $5.9 \%$ decrease in overall gray matter density $(0.251 \pm 0.031$ in CBP subjects; $0.267 \pm 0.027$ in controls; $n=17$ pairs; paired $t$ 
A

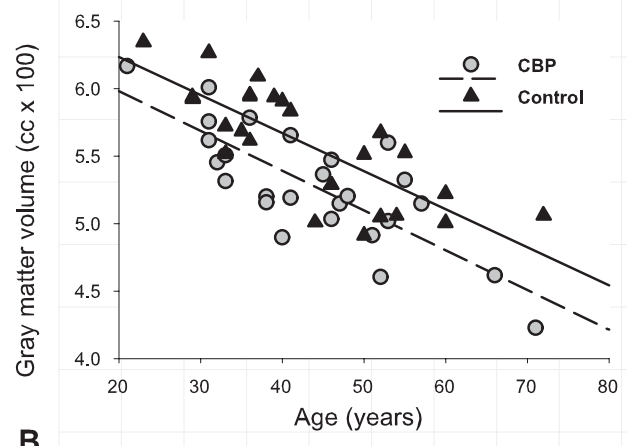

B

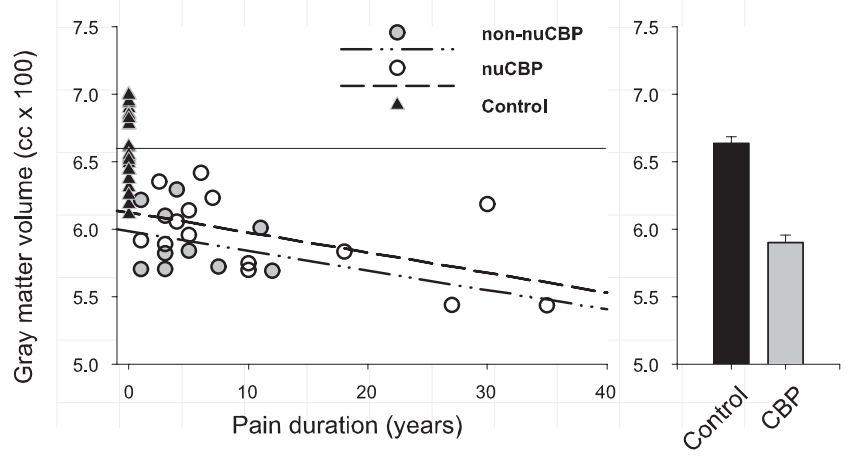

Figure 1. Decreased whole-brain cortical gray matter volume in CBP subjects. Skullnormalized neocortical gray matter volumes are shown for CBP subjects and matched control subjects. $A$, Gray matter volumes as a function of age. The difference in intercepts corresponds to an average decrease of $30 \mathrm{~cm}^{3}$ in gray matter volume in CBP compared with the normal subjects. $B$, Gray matter volumes as a function of pain duration, after correcting for age and gender. Individual control subjects are shown at pain duration $=0$. nuCBP and non-nuCBP CBP patient data are presented separately. The horizontal line is the mean volume for controls. Individual whole-brain gray matter volumes in CBP subjects are all below the mean volume for controls. Group-averaged gray matter volumes (mean \pm SEM) are shown in the right bar graph, before (top) and after (bottom) correcting for age and gender. Lines are best linear fits for each group.

test $=2.28 ; p<0.04)$. Whole-brain cortical gray matter volume correlated with age in both groups (CBP subjects, age dependence slope $=-2.9 \mathrm{~cm}^{3}, 0.5 \%$; controls, slope $=-2.8 \mathrm{~cm}^{3}$, with $r^{2}=0.6, p<0.001$ in both groups) (Fig. $1 A$ ). Thus, the ageassociated decrease in neocortical gray matter volume was 2.8 $\mathrm{cm}^{3}(0.5 \%)$ per year in both groups. This value closely agrees with previous estimates of age-dependent gray matter atrophy (Good et al., 2001a; Resnick et al., 2003).

Multiple linear regression indicated that whole-brain gray matter volume in CBP depended on age, gender, and pain duration $\left(F_{(3,20)}=28.6 ; p<10^{-5}\right)$, where pain duration had a $\beta=$ $-0.33(p<0.008)$ showing that pain duration was a significant predictor after correcting for age and gender. Consistent with previous studies (Good et al., 2001a,b), age and gender were also strong predictors of gray matter volume in normal subjects. Therefore, we corrected for these confounds and then compared the gray matter volume between controls and CBP patients (Fig. $1 B)$. The resultant gray matter volume was $663 \pm 27 \mathrm{~cm}^{3}$ in controls and $590 \pm 28 \mathrm{~cm}^{3}$ in CBP patients, reflecting an $11 \%$ decrease. Mean gray matter volume was not different between neuropathic (nuCBP) and non-neuropathic (non-nuCBP) subtypes, but dependence on pain duration was only significant in nuCBP, with corrected gray matter volume decreasing by $1.3 \mathrm{cc}$ $(0.2 \%)$ per year of chronic pain.

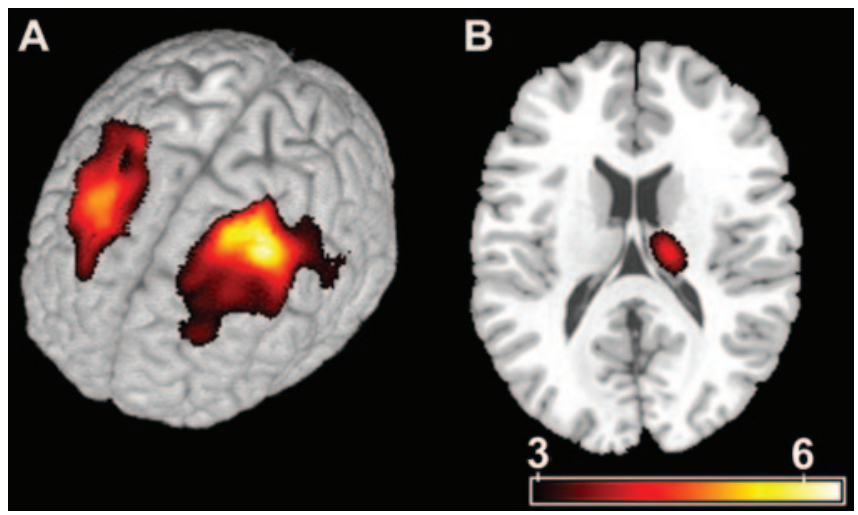

Figure 2. Regional gray matter density decreases in CBP subjects. A nonparametric comparison of voxel-based morphometry between CBP and control subjects is shown. $A$, Gray matter density is bilaterally reduced in the DLPFC. The result is from a VBM permutation-based pseudo- $t$ test and voxel-level contrasts when all brain gray matter voxels were compared between controls and CBP subjects. Pseudocolor highly positive values indicate regions where gray matter density was reduced in (BP subjects (controls - CBP). B, A nonparametric comparison spatially limited to the thalami revealed a significant decrease in gray matter density in the right anterior thalamus. A slice at the peak of decreased thalamic gray matter is shown. Pseudo-t values are color coded; range is $3-6$.

\section{Regional gray matter density}

Regional specificity of brain gray matter changes was assessed nonparametrically (Ashburner and Friston, 2000), using optimized voxel-based morphometry (Friston et al., 1995; Good et al., 2001a; Nichols and Holmes, 2002). The dorsolateral prefrontal cortex (DLPFC), bilaterally, was the main region that showed local decreased gray matter density in CBP subjects relative to controls (Fig. 2A) (supplemental Fig. 1, available at www. jneurosci.org as supplemental material). In the left hemisphere, the region contained two distinct peaks $[x, y, z$ coordinates (in millimeters), $-35,4,55$; pseudo- $t_{\max }=6.38, p=0.003$; cluster size $=25.3 \mathrm{~cm}^{3}, p=0.003$; with a second peak at $-24,5,59$; pseudo- $\left.t_{\max }=5.75, p=0.006\right]$, and a single peak was seen in the right hemisphere $\left[x, y, z, 34,18,53\right.$; pseudo- $t_{\max }=5.1, p=0.034$; cluster size $\left.=19.0 \mathrm{~cm}^{3}, p=0.005\right]$.

Because of the importance of the thalamus in mediating nociceptive inputs to the cortex, and because of repeated reports of decreases in thalamic activity in human brain imaging studies, a separate nonparametric analysis was performed for the thalamus in which the statistical comparison was performed for the space limited by the thalami. The right anterior thalamus showed a significant decrease in gray matter density in CBP subjects $[x, y, z$, $14,-18,16$; pseudo- $t_{\max }=4.3, p<0.007$; cluster size $=2.3 \mathrm{~cm}^{3}$, $p<0.01$ ] (Fig. 2B) (supplemental Fig. 2, available at www. jneurosci.org as supplemental material). The left thalamus also shows decreased gray matter density; however, this decrease did not pass cluster threshold (supplemental Fig. 2, available at www.jneurosci.org as supplemental material). No significant increases in regional gray matter density in CBP were observed.

For the three highly significant DLPFC peaks (showing the largest amount of cortical atrophy), the first eigenvariate of gray matter density was calculated, for a 5-mm-radius sphere, across subjects. Comparisons of dependence of this regional index of gray matter density on brain region (three locations) and on CBP (nuCBP, non-nuCBP, and controls) with a two-way (repeated measures for subjects) ANOVA indicated no significant differences between brain regions $(p>0.9)$ and a large difference for presence and subtype of pain $\left(F_{(2,93)}=26.5 ; p<10^{-6}\right)$. Planned comparisons between controls and CBP patients $\left(F_{(1,93)}=64.5\right.$; 


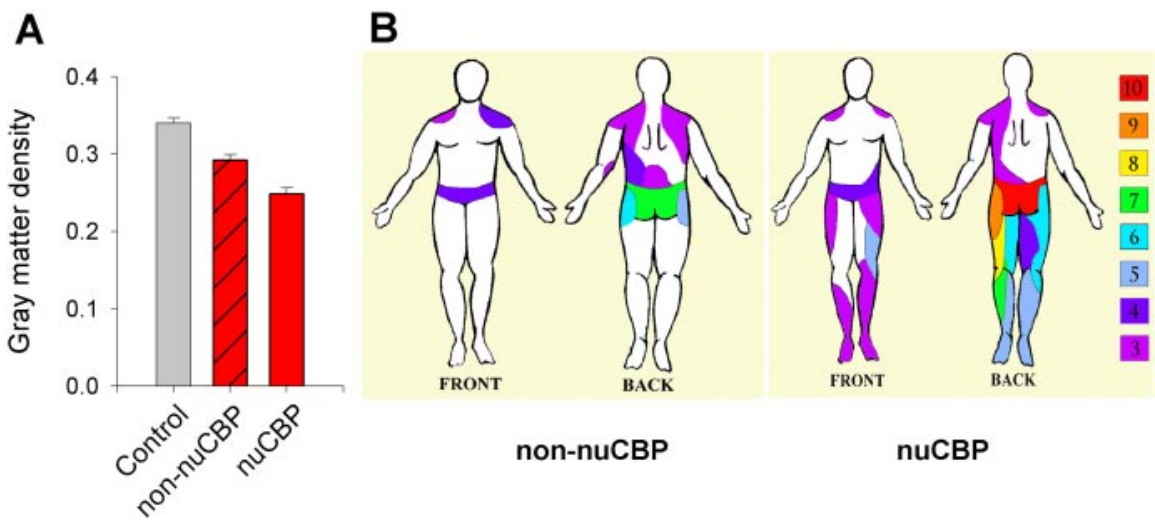

Figure 3. DLPFC gray matter density as a function of subtypes of CBP. A, DLPFC gray matter density is highest in controls and lowest in neuropathic patients. Gray matter density is in arbitrary units derived from regional eigenvariates. $B$, Somatotopy of pain is shown on the figurines to the right, for subtypes of CBP. Color code is the number of subjects localizing their pain to indicated body site. Only the 17 CBP patients used in VBM are shown. The remaining CBP patients had a similar somatotopy for CBP.

\section{Lateral ventricular volume}

We also measured skull-normalized volumes of the lateral ventricles, using a specially designed mask to isolate these structures (SIENAX analysis). There was no difference in normalized lateral ventricular volume between CBP subjects and controls (mean $\pm \mathrm{SD}$ was $21.2 \pm 8.2 \mathrm{~cm}^{3}$ in $\mathrm{CBP}$ and $20.5 \pm 7.6 \mathrm{~cm}^{3}$ in controls; paired $t$ test $=0.34 ; p>0.7)$. There were, however, significant positive correlations between lateral ventricle size in CBP and pain intensity and between sensory and negative-affective dimensions of CBP $(p<0.05)$. The change in lateral ventricle size (CBP - control) was also positively correlated with sensory and negativeaffective dimensions of CBP $(p<0.05)$.

\section{Discussion}

This is the first study showing brain mor$p<10^{-6}$ ) and between nuCBP and non-nuCBP subgroups were highly significant $\left(F_{(1,93)}=12.7 ; p<0.0006\right)$; the average decrease in DLPFC gray matter density was $14 \%$ in non-nuCBP subjects and $27 \%$ in nuCBP subjects relative to controls. Thus, within the DLPFC nuCBP patients show larger decreases in gray matter density than non-nuCBP patients (Fig. 3A).

Given that the three DLPFC regions did not differ in gray matter density, we examined their relationship to pain characteristics as a group. Across regions, DLPFC gray matter density index for all CBP, or for subtypes of CBP, was significantly or borderline significantly negatively correlated with measures directly related to pain [the intensity and duration of pain and sensory and negative-affective dimensions of CBP $(p<0.1)]$ and with age and gender but not with anxiety, depression, or drug use. In contrast, the eigenvariate for the right thalamus peak was significantly negatively correlated only with pain duration. To differentiate further the relationship between regional gray matter and pain characteristics, we subtracted the gray matter index of CBP subjects from corresponding controls. This index of change in DLPFC gray matter, which removes age and gender confounds, was regressed with pain characteristics that best distinguish CBP subjects from controls (pain intensity, duration, and sensory and affective dimensions of CBP). Across all CBP, the combination of sensory and negative-affective dimensions of CBP predicted DLPFC gray matter change $\left(F_{(2,48)}=5.3, p<\right.$ 0.007; affect $\beta=0.47, p<0.004$; sensory $\beta=-0.45, p<0.006)$. When CBP was subdivided into its subgroups, we observed differential relationships. In nuCBP subjects, pain intensity, duration, and negative affect predicted DLPFC gray matter change $\left(F_{(3,29)}=6.2, p<0.002\right.$; pain intensity $\beta=-0.64, p<0.0007$; affect $\beta=0.62, p<0.0009$ ), whereas in non-nuCBP subjects all four pain characteristics contributed to DLPFC gray matter change $\left(F_{(4,13)}=12.3, p<0.0002\right.$; pain intensity $\beta=1.48, p<$ 0.0002; pain duration $\beta=1.44, p<0.007$; affect $\beta=-1.97, p<$ $0.0018)$. Thus, regional gray matter changes are strongly and specifically related to pain characteristics, and this pattern is opposite for neuropathic compared with non-neuropathic types. This dissociation is consistent with extensive clinical data showing that neuropathic pain conditions are more debilitating and have a stronger negative affect (Dworkin, 2002), which may be directly attributable to the larger decrease in gray matter density that we observe in the DLPFC of nuCBP patients. phometric abnormalities in chronic pain. One other study examined morphometry in pain conditions (Matharu et al., 2003), in which migraine patients were contrasted to normal subjects, and no significant differences were found. We observe decreased global cortical gray matter with two independent approaches, with both assessments showing a similar amount of overall neocortical brain volume decrease in CBP. We refer to the global and regional decreases in gray matter as atrophy. This is based on the close agreement between the two measures used for estimating global gray matter decrease: the validation of VBM by manual measures and by alternate MRI measures of local gray matter decreases (Vargha-Khadem et al., 1998; Maguire et al., 2000; Richardson et al., 2004). It should be qualified, however, that only direct histological analysis can unequivocally confirm cellular atrophy. Given that normal whole-brain gray matter atrophy is $0.5 \%$ per year of aging and that atrophy caused by CBP is $5-11 \%$, the magnitude of brain gray matter atrophy caused by CBP is equivalent to 10-20 years of aging. However, this analogy only holds for the overall magnitude, because the regional specificity of atrophy in CBP is distinct from that seen with aging (Good et al., 2001a; Resnick et al., 2003).

After correcting for age and gender, individual CBP wholebrain gray matter volumes were lower than the mean of controls. Moreover, only $18 \%$ of whole-brain gray matter variance could be explained by pain duration. Therefore, a large portion of the whole-brain atrophy in $\mathrm{CBP}$ cannot be accounted for by the measured pain characteristics, implying that there may be genetic (Zubieta et al., 2003) and experiential (Perkins and Kehlet, 2000) predispositions contributing to the observed atrophy. In the DLPFC, a larger proportion of the variance could be explained by pain characteristics (40\% for nuCBP; $80 \%$ for non-nuCBP), implying a tighter relationship between regional brain atrophy and perceived pain. Therefore, we suggest that the pattern of brain atrophy is directly related to the perceptual and behavioral properties of CBP.

What does the regional pattern of atrophy imply? The observed regional pattern of atrophy is distinct from that seen in chronic depression or anxiety (Bell-McGinty et al., 2002; Almeida et al., 2003; Yamasue et al., 2003) and shows a minimal relationship with anxiety and depression traits. Thus, it seems to be specific to chronic pain, especially because the regions showing atrophy, the thalamus and DLPFC, participate in pain per- 
ception. The DLPFC is activated in acute pain, with responses that do not code stimulus intensity (Coghill et al., 1999). Recent evidence suggests that the DLPFC exerts "top-down" inhibition on orbitofrontal activity, limiting the magnitude of perceived pain (Lorenz et al., 2003). Thus, DLPFC atrophy may lead to a disruption of its control over orbitofrontal activity, which in turn is critical in the perception of negative affect in general (Small, 2002; Goel and Dolan, 2003) and particularly in pain states (Price, 2000; Apkarian et al., 2004b). Thalamic atrophy in CBP is important, because it is a major source of nociceptive inputs to the cortex (although the peak decrease in gray matter seems more anterior than the medial thalamic target of spinothalamic terminations), and damage to this region may be a reason for the generalized sensory abnormalities commonly associated with chronic pain (Moriwaki and Yuge, 1999; Rommel et al., 2001; Fishbain et al., 2003; Giesecke et al., 2004). Moreover, the thalamic atrophy that we observe provides an explanation for repeated reports of decreased baseline and stimulus-evoked activity and for abnormal chemistry within the thalamus for diverse chronic pain states (Apkarian et al., 2004b). The dorsal anterior cingulate is shown to be specifically involved in pain affect in normal subjects and exhibits decreased nociceptive signaling in various chronic pain states (Apkarian et al., 2004b), which may again be caused by thalamic atrophy because the anterior thalamus is a primary input to the anterior cingulate. Therefore, we suggest that regional atrophy dictates the brain activity observed in chronic pain, and it may explain the transition from acute to chronic pain by shifting brain activity related to pain affect away from the anterior cingulate to orbitofrontal cortex.

It is possible that some of the observed decreased gray matter reflects tissue shrinkage (changes in extracellular space and microvascular volume may cause tissue shrinkage without substantially impacting neuronal properties), implying that proper treatment would reverse this portion of the decreased brain gray matter. The atrophy may be also attributable to more irreversible processes, such as neurodegeneration, which we favor because the main brain region involved (the DLPFC) also exhibits decreased $N$-acetyl-aspartate (Grachev et al., 2000), and decreased $\mathrm{N}$-acetyl-aspartate has been observed in most neurodegenerative conditions, implying that it may be a marker for cell density in the brain (Salibi and Brown, 1998), and because spinal cord neurons undergo apoptosis in rats with neuropathic pain (Whiteside and Munglani, 2001; Moore et al., 2002; de Novellis et al., 2004). Similarly, within the thalamus, abnormal chemistry and decreased baseline and stimulus-evoked activity are all consistent with the notion that this region may undergo atrophy. We do not know the specific elements within the thalamus and DLPFC undergoing atrophy; and whether they involve projecting neurons, interneurons, or both, as well as glia, and in what proportions. In the spinal cord, the evidence suggests that mainly GABAergic inhibitory interneurons undergo apoptosis. Extrapolating from this evidence, we assume that at the supraspinal level as well, atrophy impinges primarily on interneurons. Recent evidence also suggests that after nerve injury, some components of pain behavior are a consequence of hyperactivity of spinal cord microglia (Tsuda et al., 2003), and a histological study has shown a reduction in glial numbers in the cortex in major depressive disorder and bipolar disorder (Ongur et al., 1998). Thus, changes in glial numbers may be important in the atrophy we observe in CBP as well; this remains to be determined. Because the relationship between DLPFC gray matter decrease and pain parameters is distinct for subtypes of CBP, it is likely that the extent of involvement of different cellular types will also vary with type of chronic pain.

Independent methods were used for determining global brain gray matter volume to increase our confidence with the observed results. We also used nonparametric analysis of VBM mainly because this circumvents Gaussian distribution assumptions necessary for proper calculation of cluster threshold in parametric VBM, and because the approach directly compensates for multiple-comparison correction. We controlled for drug consumption in CBP by using a unitary scale, which showed no relationship to global or local measures of gray matter. However, the implications of this negative result are not clear, because the tool we used (MQS) reduces multiple drugs with multiple doses to a unitary scale and thus may obscure effects of some drugs by others. More-specific drug effects on brain gray matter might be uncovered if drugs used by CBP patients are parceled into separate categories and then related to brain morphometry. Such a study would require a much larger cohort (we do not have enough subjects in any particular category in the current study). We should also state that the current study was cross-sectional in design, which can uncover relationships but not establish causality. To achieve the latter, a longitudinal design is necessary. Our results indicate that the type of CBP is relevant to brain atrophy. In future studies, it will be important to delineate clinical syndromes of CBP into multiple more-homogeneous categories, because this may further parcel the location and degree of changes of brain volume and density.

Given that, by definition, chronic pain is a state of continuous persistent perception with associated negative affect and stress, a parsimonious mechanistic explanation for the decreased gray matter is overuse atrophy caused by excitotoxicity and inflammatory agents (Brown and Bal-Price, 2003; Mattson, 2003), potentiated by predisposing factors. We cannot rule out the contribution of possible lifestyle differences between the patients and control subjects to the observed differences in gray matter. However, we can assert the coexistence of theoretically independent effects (i.e., group differences in global and local brain gray matter volumes) determined by two independent methods (the association between global and regional densities with pain duration within the patient group and the association between regional densities and pain parameters within the patient group that also distinguishes between subtypes of CBP) that provide compelling evidence for the importance of the observed morphometric changes in the pathophysiology of CBP. We hypothesize that atrophy of the brain circuitry involved in pain perception may dictate the properties of the pain state, such that as atrophy of elements of the circuitry progresses, the pain condition becomes more irreversible and less responsive to therapy.

\section{References}

Almeida OP, Burton EJ, Ferrier N, McKeith IG, O’Brien JT (2003) Depression with late onset is associated with right frontal lobe atrophy. Psychol Med 33:675-681.

Apkarian AV, Sosa Y, Krauss BR, Thomas PS, Fredrickson BE, Levy RE, Harden R, Chialvo DR (2004a) Chronic pain patients are impaired on an emotional decision-making task. Pain 108:129-136.

Apkarian AV, Bushnell MC, Treede RD, Zubieta JK (2004b) Human brain mechanisms of pain perception and regulation in health and disease. Eur J Pain, in press.

Ashburner J, Friston KJ (2000) Voxel-based morphometry-the methods. NeuroImage 11:805-821.

Beck AT, Steer RA (1993a) Beck depression inventory. San Antonio, TX: Psychological Corporation.

Beck AT, Steer RA (1993b) Manual for the Beck anxiety inventory. San Antonio, TX: Psychological Corporation. 
Bell-McGinty S, Butters MA, Meltzer CC, Greer PJ, Reynolds III CF, Becker JT (2002) Brain morphometric abnormalities in geriatric depression: long-term neurobiological effects of illness duration. Am J Psychiatry 159:1424-1427.

Brown GC, Bal-Price A (2003) Inflammatory neurodegeneration mediated by nitric oxide, glutamate, and mitochondria. Mol Neurobiol 27:325-355.

Cavanaugh JM, Weinstein JN (1994) Low back pain: epidemiology, anatomy and neurophysiology. In: Textbook of pain (Wall PD, Melzack R, eds), pp 441-456. Edinburgh: Churchill Livingstone.

Coghill RC, Sang CN, Maisog JM, Iadarola MJ (1999) Pain intensity processing within the human brain: a bilateral, distributed mechanism. J Neurophysiol 82:1934-1943.

de Novellis V, Siniscalco D, Galderisi U, Fuccio C, Nolano M, Santoro L, Cascino A, Roth KA, Rossi F, Maione S (2004) Blockade of glutamate mGlu5 receptors in a rat model of neuropathic pain prevents early overexpression of pro-apoptotic genes and morphological changes in dorsal horn lamina II. Neuropharmacology 46:468-479.

Deyo RA (1998) Low-back pain. Sci Am 279:48-53.

Deyo RA, Weinstein JN (2001) Low back pain. N Engl J Med 344:363-370.

Dworkin RH (2002) An overview of neuropathic pain: syndromes, symptoms, signs, and several mechanisms. Clin J Pain 18:343-349.

Fishbain DA, Cutler RB, Lewis J, Cole B, Rosomoff RS, Rosomoff HL (2003) Is the location of nondermatomal sensory abnormalities (NDSAs) related to pain location? Pain Med 4:238-243.

Friston KJ, Holmes AP, Worsley KJ, Poline J-B, Frith CD, Frackowiak RS (1995) Statistic parametric maps in functional imaging: a general linear approach. Hum Brain Mapp 2:189-210.

Giesecke T, Gracely RH, Grant MA, Nachemson A, Petzke F, Williams DA, Clauw DJ (2004) Evidence of augmented central pain processing in idiopathic chronic low back pain. Arthritis Rheum 50:613-623.

Goel V, Dolan RJ (2003) Reciprocal neural response within lateral and ventral medial prefrontal cortex during hot and cold reasoning. NeuroImage 20:2314-2321.

Good CD, Johnsrude IS, Ashburner J, Henson RN, Friston KJ, Frackowiak RS (2001a) A voxel-based morphometric study of ageing in 465 normal adult human brains. NeuroImage 14:21-36.

Good CD, Johnsrude I, Ashburner J, Henson RN, Friston KJ, Frackowiak RS (2001b) Cerebral asymmetry and the effects of sex and handedness on brain structure: a voxel-based morphometric analysis of 465 normal adult human brains. NeuroImage 14:685-700.

Grachev ID, Fredrickson BE, Apkarian AV (2000) Abnormal brain chemistry in chronic back pain: an in vivo proton magnetic resonance spectroscopy study. Pain 89:7-18.

Harstall C, Ospina M (2003) How prevalent is chronic pain? Pain Clinical Updates 11:1-4.

Hunt SP, Mantyh PW (2001) The molecular dynamics of pain control. Nat Rev Neurosci 2:83-91.

Julius D, Basbaum AI (2001) Molecular mechanisms of nociception. Nature 413:203-210.

Lorenz J, Minoshima S, Casey KL (2003) Keeping pain out of mind: the role of the dorsolateral prefrontal cortex in pain modulation. Brain 126:1079-1091.

Maguire EA, Gadian DG, Johnsrude IS, Good CD, Ashburner J, Frackowiak RS, Frith CD (2000) Navigation-related structural change in the hippocampi of taxi drivers. Proc Natl Acad Sci USA 97:4398-4403.

Matharu MS, Good CD, May A, Bahra A, Goadsby PJ (2003) No change in the structure of the brain in migraine: a voxel-based morphometric study. Eur J Neurol 10:53-57.

Mattson MP (2003) Excitotoxic and excitoprotective mechanisms: abundant targets for the prevention and treatment of neurodegenerative disorders. Neuromolecular Med 3:65-94.
Melzack R (1987) The short-form McGill pain questionnaire. Pain 30:191-197.

Mendell JR, Sahenk Z (2003) Clinical practice. Painful sensory neuropathy. N Engl J Med 348:1243-1255.

Merskey H, Bogduk N (1994) Classification of chronic pain: descriptions of chronic pain syndromes and definitions of pain terms, Ed 2. Seattle: International Association for the Study of Pain.

Middaugh SJ, Masters SL, Kee WG (1987) The medication quantification scale: A method of quantifying medication use in chronic pain patients. Biofeedback Self Regul 12:157-158.

Moore KA, Kohno T, Karchewski LA, Scholz J, Baba H, Woolf CJ (2002) Partial peripheral nerve injury promotes a selective loss of GABAergic inhibition in the superficial dorsal horn of the spinal cord. J Neurosci 22:6724-6731.

Moriwaki K, Yuge O (1999) Topographical features of cutaneous tactile hypoesthetic and hyperesthetic abnormalities in chronic pain. Pain 81:1-6.

Nichols TE, Holmes AP (2002) Nonparametric permutation tests for functional neuroimaging: a primer with examples. Hum Brain Mapp 15:1-25.

Ongur D, Drevets WC, Price JL (1998) Glial reduction in the subgenual prefrontal cortex in mood disorders. Proc Natl Acad Sci USA 95:13290-13295.

Perkins FM, Kehlet H (2000) Chronic pain as an outcome of surgery. A review of predictive factors. Anesthesiology 93:1123-1133.

Price DD (2000) Psychological and neural mechanisms of the affective dimension of pain. Science 288:1769-1772.

Resnick SM, Pham DL, Kraut MA, Zonderman AB, Davatzikos C (2003) Longitudinal magnetic resonance imaging studies of older adults: a shrinking brain. J Neurosci 23:3295-3301.

Richardson MP, Strange BA, Dolan RJ (2004) Encoding of emotional memories depends on amygdala and hippocampus and their interactions. Nat Neurosci 7:278-285.

Riley JL, Robinson ME, Wade JB, Myers CD, Price DD (2001) Sex differences in negative emotional responses to chronic pain. J Pain 2:354-359.

Rommel O, Malin JP, Zenz M, Janig W (2001) Quantitative sensory testing, neurophysiological and psychological examination in patients with complex regional pain syndrome and hemisensory deficits. Pain 93:279-293.

Salibi N, Brown MA (1998) Clinical MR spectroscopy. New York: Wiley.

Small DM (2002) Toward an understanding of the brain substrates of reward in humans. Neuron 33:668-671.

Smith SM, Zhang Y, Jenkinson M, Chen J, Matthews PM, Federico A, De Stefano N (2002) Accurate, robust, and automated longitudinal and cross-sectional brain change analysis. NeuroImage 17:479-489.

Tsuda M, Shigemoto-Mogami Y, Koizumi S, Mizokoshi A, Kohsaka S, Salter MW, Inoue K (2003) P2X4 receptors induced in spinal microglia gate tactile allodynia after nerve injury. Nature 424:778-783.

Vargha-Khadem F, Watkins KE, Price CJ, Ashburner J, Alcock KJ, Connelly A, Frackowiak RS, Friston KJ, Pembrey ME, Mishkin M, Gadian DG, Passingham RE (1998) Neural basis of an inherited speech and language disorder. Proc Natl Acad Sci USA 95:12695-12700.

Whiteside GT, Munglani R (2001) Cell death in the superficial dorsal horn in a model of neuropathic pain. J Neurosci Res 64:168-173.

Woolf CJ, Salter MW (2000) Neuronal plasticity: increasing the gain in pain. Science 288:1765-1769.

Yamasue H, Kasai K, Iwanami A, Ohtani T, Yamada H, Abe O, Kuroki N, Fukuda R, Tochigi M, Furukawa S, Sadamatsu M, Sasaki T, Aoki S, Ohtomo K, Asukai N, Kato N (2003) Voxel-based analysis of MRI reveals anterior cingulate gray-matter volume reduction in posttraumatic stress disorder due to terrorism. Proc Natl Acad Sci USA 100:9039-9043.

Zubieta JK, Heitzeg MM, Smith YR, Bueller JA, Xu K, Xu Y, Koeppe RA, Stohler CS, Goldman D (2003) COMT val158met genotype affects mu-opioid neurotransmitter responses to a pain stressor. Science 299: $1240-1243$. 